\title{
Artropofauna edáfica asociada a cultivo de Plátano Musa x paradisiaca L. Dominico-Hartón Musa AAB Simmons (MUSACEAE) en Calarcá, Quindío
}

\section{Arthropofauna of soil associated with cultivation Dominico Hartón (Musa AAB) in Calarcá, Quindío}

\author{
Molina Rico Ligia Janneth ${ }^{1 *}$ M.Sc, Arana Castañeda Carlos Andrés ${ }^{1}$ Lic, \\ Landázuri Patricia ${ }^{2}$ Ph.D, Girón Vanderhuck Mercedes ${ }^{1}$ Ph.D. \\ ${ }^{1}$ Universidad del Quindío, Facultad de Educación, Grupo de Investigación BIOEDUQ. Colombia. \\ ${ }^{2}$ Universidad del Quindío, Facultad de Ciencias de la Salud, grupo de Investigacion GECAVYME. Colombia.
}

\section{Keywords:}

Community; agro-production; agrochemical; organic amendments.

\begin{abstract}
In this paper we analyze the edaphic arthropod community structure associated to a secondary forest in Calarcá-Quindío, and a plantain crop under organic amendments (Necromasa, Compost) and under agrochemicals, with two planting distances between plant and plant $(1.7 \mathrm{~m}$ and $2.5 \mathrm{~m})$, between June 2013 and January 2014, under the methodology proposed by the Programme Tropical Soil Biology and Fertility; during the analysed period were taken three (3) soil monoliths every month. Were collected 18929 individuals distributed in 52 families of insects, 16 of millipedes, 8 of arachnids and 1 crustaceans. Sampling effort is represented by ACE and Chao 1 estimators higher than $82 \%$ for both coverages (crop and forest). In general, there were no differences in the community structure due to the treatments. Nevertheless the arachnid and myriapod communities were substantially affected, while insects were altered by the crop microclimate variation. It is concluded that the type of fertilization and planting distances do not affect the soil arthropods, but modifies the soil microclimatic and biological conditions.
\end{abstract}

\section{Resumen}

En este trabajo se analiza la estructura de la comunidad de artrópodos edáficos asociados a un bosque secundario (Calarcá-Quindío), y a un cultivo de plátano bajo enmiendas orgánicas (Necromasa, Compost) y bajo agroquímicos, con dos distancias de siembra entre planta y planta $(1,7 \mathrm{~m}$ y $2,5 \mathrm{~m})$, en los meses de junio 2013 a enero 2014. Mensualmente se tomaron 3 monolitos de suelo por cada tratamiento bajo la metodología propuesta por el Programa de Biología y Fertilidad de los Suelos Tropicales. 18.929 individuos fueron colectados, distribuidos en 52 familias de insectos, 16 de miriápodos, 8 de arácnidos y 1 de crustáceos. Se evidencia un esfuerzo de muestreo con estimadores ACE y CHAO1 superior al $82 \%$ en las dos coberturas (cultivo y bosque). De manera general no se presentaron diferencias en la estructura de la comunidad a causa de los tratamientos pero se observa que estos modifican sustancialmente los grupos de arácnidos y miriápodos; mientras que los insectos parecen ser impactados por la variación microclimática del cultivo. Se concluye que los tipos de fertilización y las distancias de siembra no afectan la artropofauna edáfica; aunque se evidencia la modificación en las condiciones microclimáticas y biológicas del suelo por lo tratamientos. 


\section{Introducción}

Los ecosistemas terrestres y los sistemas agrícolas se sustentan sobre el sistema suelo, la biodiversidad que se observa en este sustrato depende de las interacciones que suceden en su interior. De igual forma las civilizaciones pasadas, presentes y futuras han construido y construirán sobre el recurso edáfico su desarrollo social y económico (RITZ y VAN DER PUTTEN, 2012). Actualmente, existe gran preocupación por el manejo y conservación del suelo y su impacto en la dinámica cultural, ambiental, socioeconómica y de sustentabilidad de las naciones, dado los crecientes problemas de transformación que afectan a nivel global dicho sistema.

La región Andina no es ajena a estos procesos de transformación de la dinámica ecológica del suelo por la introducción de cultivos, particularmente café y plátano. En los últimos 30 años en el eje cafetero colombiano el panorama del uso del terreno ha cambiado drásticamente debido a la baja de los precios del café, la sobreoferta en el mercado internacional y los problemas fitosanitarios. Las nuevas alternativas introducidas conllevaron a la reducción o desaparición, en algunas localidades, de los cultivares de café tradicional asociados con plátano o sembrados con variedades mejoradas y se transformaron en cultivos industrializados o mixtos con plátano para lograr opciones que brindaran bienestar económico a los cultivadores (ZUÑIGA et al., 2013).

De acuerdo con LAVELLE et al., (2012), los suelos albergan comunidades muy abundantes y diversas de artropofauna. Estos organismos crean hábitats cavando el suelo, mezclándolo y fragmentando los residuos vegetales. Estos seres han desarrollado funciones complementarias para digerir los recursos alimenticios de baja calidad y se han adaptado a vivir en suelos compactados con humedad inestable. Los cultivos y los agroquímicos empleados comúnmente afectan gravemente el suelo y la fauna edáfica, mientras que los insumos orgánicos y una diversidad mínima de comunidades vegetales a escala local ayudan a mantener la salud del suelo y de los ecosistemas productivos, lo que genera un servicio ecosistémico.
De esta manera, la transformación de zonas boscosas a sistemas de producción agrícola tiene efectos irreversibles sobre las comunidades de artrópodos edáficos, suscitado por el agotamiento de recursos orgánicos mixtos que favorecen el sostenimiento de dichas comunidades, y a su vez la fertilidad y sostenibilidad del sistema suelo (FEIJOO et al., 2007; FEIJOO et al., 2010). La importancia de la artropofauna edáfica se relaciona con la regulación de la dinámica de la materia orgánica, el ciclo biogeoquímico de nutrientes y en la estructura del suelo (COLEMAN et al., 2004, DECAENS et al., 2003, FEIJOO et al., 2007, LAVELLE 2012). Estos procesos son esenciales para el funcionamiento del ecosistema y sustentan la producción agrícola.

Para el caso, la artropofauna genera una respuesta rápida a los cambios en el manejo del suelo reflejados en la presencia o ausencia en algunos grupos tales como Diplopoda, Blattaria, entre otros. La relación entre los grupos de la artropofauna del suelo y las diferentes prácticas de fertilización ha sido motivo de constante preocupación, aunque se ha prestado poca atención a la respuesta de éste tipo de fauna a los cambios en la fertilidad. La evaluación de la comunidad de artropofauna en suelos de cultivo de plátano sometido a diferentes prácticas de fertilización es de vital importancia, con el fin de observar los efectos de las condiciones ambientales en la estructura comunitaria. Por lo cual, el objetivo de esta investigación fue comparar la estructura de la comunidad de artropofauna edáfica encontrada en un bosque y la asociada a un cultivo de plátano Dominico Hartón (Musa AAB) sometido a diferentes prácticas de fertilización en Calarcá, Quindío y ver si estos manejos de cultivo afectan la comunidad de artropofauna.

\section{Materiales y métodos}

Área de estudio. El trabajo se realizó en la finca "La Bella Nubia" (FBN) (4²9'48.8" N 7540'20." O), y en el Jardín Botánico del Quindío (JBQ) (4³1'57" N y 75³8'37' O) en Calarcá-Quindío, entre los 1492 y 1497 m.s.n.m, con temperatura media de $20^{\circ} \mathrm{C}$, precipitación anual de $2000 \mathrm{~mm}$ y humedad relativa del 85\% (CRQ 2014). La FBN se caracteriza por presentar monocultivo de Plátano en el $75 \%$ de sus predios (3 ha aprox.). 
EI JBQ es un bosque secundario dominado por bosque muy húmedo premontano (bmh-PM) y selva subandina, con un relieve ondulado y plano (HOLDRIDGE, 1987).

Diseño experimental. El cultivo de plátano de la FBN desde su inicio fue sometido a un tipo de fertilización (Agroquímico [A]), dos tipos de enmiendas orgánicas (Compost [C] y Necromasa $[\mathrm{N}]$ ), parcelas testigo (T) y dos distancias de siembra entre planta y planta ([1]: $1,7 \mathrm{~m}-[2]$ : $2,5 \mathrm{~m})$. La composición y aplicación del $\mathrm{A}$ y de las enmiendas orgánicas fueron: $A$ Platanero $®$ (Nitrógeno 14, Fósforo 4, Potasio 23, Magnesio 4 , Calcio y menores 4) con una cantidad de $120 \mathrm{~g} /$ planta; C derivado de lombricultivo con un total de $1,5 \mathrm{~kg} /$ planta; $\mathrm{N}$ (hoja y pseudotallo de Plátano picado) con una cantidad de $1,5 \mathrm{~kg} /$ planta; y parcelas $T$ plantas sin ningún tipo de enmienda o fertilización seleccionadas al azar (Tabla 1). Se contó con 8 tratamientos divididos en 96 parcelas instaladas al azar con un total de 12 parcelas/ tratamiento.

Los tratamientos corresponden a cada tipo de manejo por cada una de las distancias entre planta y planta (Tabla 1). El tamaño de las parcelas no es homogéneo ya que varía según las distancias utilizadas. El bosque secundario (B) del JBQ fue utilizado como control natural con el fin de comparar estructura de la comunidad de artrópodos edáficos con la del cultivo de plátano de la FBN. Para esto se evaluaron tres monolitos mensuales, debido a que la FBN ha sido sometida a antiguos procesos de agro-producción.

Tabla 1. Tratamientos asignados y nomenclatura empleada

\begin{tabular}{cc}
\hline Nomenclatura & Tratamiento/distancia \\
\hline A1 & Agroquímico/1,7m \\
A2 & Agroquímico/2,5m \\
N1 & Necromasa $/ 1,7 \mathrm{~m}$ \\
N2 & Necromasa $/ 2,5 \mathrm{~m}$ \\
C1 & Compost $/ 1,7 \mathrm{~m}$ \\
C2 & Compost $/ 2,5 \mathrm{~m}$ \\
T1 & Sin fertilización $/ 1,7 \mathrm{~m}$ \\
T2 & Sin fertilización $/ 2,5 \mathrm{~m}$ \\
B & Bosque JBQ \\
\hline
\end{tabular}

Colecta e identificación de artrópodos edáficos. Para el muestreo de artropofauna edáfica se empleó la metodología del monolito, recomendada por el Instituto de Fertilidad y Biología de
Suelos Tropicales (TSBF); cada monolito define un volumen de suelo de $25 \times 25 \times 20 \mathrm{~cm}$ de profundidad, el cual se divide en cuatro estratos; hojarasca, $0-10 \mathrm{~cm}, 10-20 \mathrm{~cm}$ y $20-$ $30 \mathrm{~cm}$ (ANDERSON e INGRAM, 1993). En cada tratamiento y control se tomaron mensualmente 3 monolitos de suelo de manera simultánea, durante 8 meses (junio 2013 - enero 2014). Se registraron las siguientes variables: temperatura ambiental y del suelo determinada con un termómetro ambiental y de suelo; humedad relativa del aire registrada con un termo-higrómetro y la humedad relativa del suelo estimada a partir de la diferencia entre el peso húmedo y el peso seco de tres muestras de $100 \mathrm{~g}$ de suelo, secadas en una estufa de aire forzado a $60^{\circ} \mathrm{C}$ por 24 horas.

Los artrópodos se separaron manualmente de acuerdo al tamaño corporal $>2 \mathrm{~mm}$ (SWIFT et al., 1979), fueron preservados en solución de alcohol al $70 \%$ y determinados tanto larvas como adultos hasta familia a partir del empleo de claves taxonómicas (DELVARE et al., 2002; BORROR and WHITE, 1970; CHOATE, 2010; GIBB and OSETO, 2006; WOLFF, 2006) y la comparación con la colección de artrópodos del Museo de Artrópodos de la Universidad del Quindío en Armenia, Quindío. El esfuerzo y efectividad de muestreo se evaluó por medio de curvas de acumulación de especies y los estimadores de la riqueza esperada ACE y Chao 1 utilizando el programa EstimateS 5.0.1 con 100 aleatorizaciones (COLWELL, 1997).

Análisis de los datos. El esfuerzo y efectividad de muestreo se evaluó por medio de curvas de acumulación de especies y los estimadores de la riqueza esperada ACE y Chao 1 utilizando EstimateS 5.0.1 con 100 aleatorizaciones (COLWELL, 1997). Se construyeron las variables comunitarias de estudio: Abundancia por familia, Riqueza de familias, Diversidad del exponencial de índice Shannon-Wiever (exp Shannon) (LOU y GONZÁLEZ, 2012), Dominancia de Simpson y Equidad o uniformidad de Pielou. Posteriormente se verificaron los requisitos de homogeneidad y normalidad de los datos (Kolmogorov, Smirnov y Bartlet), seguido de un análisis de ANOVA y de diferencias mínimas significativas entre las variables de comunidad, determinadas por los factores evaluados (tratamientos y tiempo de muestreo). Finalmente se realizó un análisis de 
componentes principales (ACP) con el fin de comparar los atributos ecológicos de la comunidad de artrópodos edáficos según los tipos de manejo (tratamientos y control) utilizando el software Infostat $\circledast(2016)$.

\section{Resultados}

Se colectaron 18.929 individuos, de los cuales 18.371 son insectos distribuidos en 52 familias (Tabla 2), 356 miriápodos en 16 familias (Tabla 3) y 195 arácnidos en 8 familias (Tabla 4); los crustáceos no fueron incluidos en los análisis debido a la abundancia y riqueza baja (7 individuos Oniscidae). La familia más abundante fue Formicidae con el $80 \%$ de los individuos, seguida por Scarabaeidae y Cydnidae con el $3,4 \%$ y el $2,7 \%$ respectivamente.
Tabla 3. Abundancia y riqueza de miriápodos edáficos asociados a cultivo de Plátano.

\begin{tabular}{lcc}
\hline \multicolumn{1}{c}{ Familia } & Ab. & $\begin{array}{c}\text { Presencial } \\
\text { Tipo de manejo }\end{array}$ \\
\hline Geophilidae & 172 & Todos \\
Ballophilidae & 1 & B \\
Oryidae & 2 & B \\
Mecistocephalidae & 1 & B \\
Henicopidae & 25 & A2, C1, C2, N1, N2, T1, T2 \\
Cryptopidae & 6 & B \\
Scolopocryptopidae & 14 & B \\
Scolopendridae & 1 & B \\
Rhinocricidae & 58 & Todos \\
Spirobolida (I.N.I) & 6 & A1, C1, T1, T2 \\
Spirostreptidae & 29 & Todos \\
Pseudonannolenidae & 4 & B \\
Fhurmmanodesmidae & 6 & A1, N1, T2, B \\
Aphelidesmidae & 1 & B \\
Chelodesmidae & 15 & C2, N2, B \\
Cryptodesmidae & 5 & C1, C2, T1, B \\
Polydesmida (I.N.I) & 5 & A2, C1, T2, B \\
Stemmiulidae & 5 & B \\
\hline \multicolumn{1}{c}{ Total } & 356 &
\end{tabular}

A1: AGD1; A2: AGD2; C1: COD1; C2: COD2; N1: NED1; N2: NED2; T1: TD1; T2: TD2; B: Bosque. I.N.I: Inmaduro no identificado. Ab: Abundancia.

Tabla 2. Abundancia y riqueza de insectos edáficos asociados a cultivo de Plátano.

\begin{tabular}{|c|c|c|c|c|c|}
\hline Familia & Ab. & Presencia/ Tipo de manejo & Familia & Ab. & Presencial Tipo de manejo \\
\hline Formicidae & 15113 & Todos & Scydmaenidae & 9 & $\mathrm{~N} 2, \mathrm{~T} 1, \mathrm{~B}$ \\
\hline Figitidae & 15 & $\mathrm{~A} 1, \mathrm{~A} 2, \mathrm{C} 1, \mathrm{C} 2, \mathrm{~N} 1, \mathrm{~T} 2$ & Trogositidae & 1 & $\mathrm{~A} 2$ \\
\hline Halictidae & 2 & $\mathrm{C} 1$ & Dascillidae & 1 & $\mathrm{~T} 1$ \\
\hline Braconidae & 2 & $\mathrm{C} 2, \mathrm{~N} 2$ & Carabidae & 132 & Todos \\
\hline Eulophidae & 2 & B & Lampyridae & 3 & $\mathrm{~A} 1, \mathrm{C} 1, \mathrm{C} 2$ \\
\hline Rhinotermitdae & 141 & B & Ptillidae & 3 & $\mathrm{~A} 1, \mathrm{C} 2, \mathrm{~T} 1$ \\
\hline Cimicidae & 1 & B & Sciaridae & 2 & $\mathrm{~T} 1$ \\
\hline Cicadidae & 2 & $\mathrm{~B}$ & Mydidae & 1 & $\mathrm{C} 2$ \\
\hline Cydnidae & 512 & Todos & Muscidae & 1 & $\mathrm{~N} 1$ \\
\hline Gelastocoridae & 55 & $\mathrm{~A} 1, \mathrm{~A} 2, \mathrm{C} 1, \mathrm{C} 2, \mathrm{~N} 1, \mathrm{~N} 2, \mathrm{~T} 1, \mathrm{~T} 2$ & Chironomidae & 16 & $\mathrm{~A} 1, \mathrm{C} 1, \mathrm{C} 2, \mathrm{~N} 1, \mathrm{~T} 1, \mathrm{~T} 2, \mathrm{~B}$ \\
\hline Cicadellidae & 11 & A1, A2, C1, N1, N2, T1, T2 & Calliphoridae & 2 & $\mathrm{~N} 2$ \\
\hline Lygaeidae & 55 & $\mathrm{~A} 1, \mathrm{~A} 2, \mathrm{C} 1, \mathrm{C} 2, \mathrm{~N} 1, \mathrm{~N} 2, \mathrm{~T} 1, \mathrm{~T} 2$ & Drosophlidae & 3 & $\mathrm{~A} 1, \mathrm{~A} 2, \mathrm{~N} 1$ \\
\hline Reduviidae & 2 & $\mathrm{~A} 1, \mathrm{~B}$ & Phoridae & 1 & $\mathrm{~T} 1$ \\
\hline Pseudococcidae & 2 & T2 & Asilidae & 5 & $\mathrm{~T} 2, \mathrm{~B}$ \\
\hline Labiidae & 207 & Todos & Diptera (I.N.I) & 10 & $\mathrm{C} 1, \mathrm{C} 2, \mathrm{~N} 1, \mathrm{~N} 2, \mathrm{~T} 1, \mathrm{~T} 2, \mathrm{~B}$ \\
\hline Blattellidae & 18 & $\mathrm{~A} 1, \mathrm{~A} 2, \mathrm{C} 1, \mathrm{~N} 2, \mathrm{~T} 1, \mathrm{~B}$ & Pyrallidae & 332 & Todos \\
\hline Blaberidae & 5 & $\mathrm{~A} 1, \mathrm{C} 1$ & Noctuidae & 5 & A2, C1, C2, N1, T2 \\
\hline Blattidae & 1 & N2 & Lepidoptera (I.N.I) & 22 & $\mathrm{~A} 1, \mathrm{~A} 2, \mathrm{~N} 1, \mathrm{~N} 2, \mathrm{~T} 1, \mathrm{~T} 2, \mathrm{~B}$ \\
\hline Scarabaeidae & 644 & Todos & Paronellidae & 2 & B \\
\hline Anthicidae & 2 & $A 1, B$ & Gryllidae & 32 & Todos \\
\hline Tenebrionidae & 64 & Todos & Gryllacrididae & 1 & B \\
\hline Coccinellidae & 12 & $\mathrm{~A} 1, \mathrm{~A} 2, \mathrm{C} 1, \mathrm{C} 2, \mathrm{~N} 2, \mathrm{~T} 1, \mathrm{~T} 2$ & Gryllotalpidae & 20 & $\mathrm{~A} 1, \mathrm{~N} 1, \mathrm{~T} 2$ \\
\hline Cleridae & 2 & $\mathrm{~T} 2$ & Pulicidae & 129 & C2, B \\
\hline Pselaphidae & 3 & B & Japygidae & 56 & Todos \\
\hline Chrysomellidae & 47 & Todos & Ascalaphidae & 1 & A1 \\
\hline Nitidullidae & 2 & B & Insecta (I.N.I) & 4 & $\mathrm{C} 1, \mathrm{~N} 2, \mathrm{~B}$ \\
\hline Curculionidae & 37 & Todos & & & \\
\hline Elateridae & 349 & Todos & & & \\
\hline Staphylinidae & 269 & Todos & TOTAL & 18371 & \\
\hline
\end{tabular}


Tabla 4. Abundancia y riqueza de arácnidos edáficos asociados a cultivo de Plátano.

\begin{tabular}{lcc}
\hline \multicolumn{1}{c}{ Familia } & Ab. & Presencia/ Tipo de manejo \\
\hline Araneidae & 43 & A1, A2, C1, C2, N1, N2, T1, B \\
Lycosidae & 10 & A1, A2, C1, N1, N2, T1, T2 \\
Gnaphosidae & 34 & A1, C1, C2, N1, N2, T1, T2, B \\
Pholcidae & 1 & B \\
Araneae (I.N.I) & 1 & T1 \\
Hubbardiidae & 2 & B \\
Chernetidae & 4 & C1, B \\
Zalmoxidae & 12 & A2, B \\
Oribatida (I.N.I) & 7 & A2, N1, N2, T2 \\
Parasitidae & 81 & Todos \\
\hline \multicolumn{1}{c}{ Total } & 195 & \\
\hline ATAGD A2:AGD2;
\end{tabular}

A1: AGD1; A2: AGD2; C1: COD1; C2: COD2; N1: NED1; N2: NED2; T1: TD1; T2: TD2; B: Bosque. I.N.I: Inmaduro no identificado. Ab: Abundancia.

El muestreo fue significativo para el área y el tiempo de estudio con porcentajes de confiabilidad del $87,44 \%$ y $92,31 \%$ para los estimadores ACE y CHAO1, respectivamente (Figura 1). Los datos no presentan normalidad $u$ homogeneidad $(p=0,0001)$, por lo cual los análisis estadísticos se realizaron a través de la prueba no paramétrica de Kruskal Wallis.

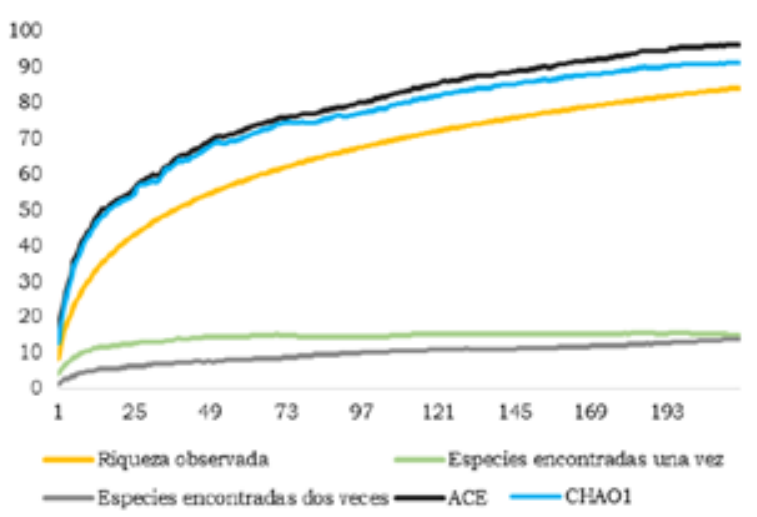

Figura 1.Curva de acumulación de especies según los estimadores de riqueza $\mathrm{ACE}$ y $\mathrm{CHAO} 1$ para el sitio de muestreo.

Mes a mes se colectaron en el cultivo de plátano FBN, entre 7 y 9 taxones de insectos, 5 a 7 taxones de miriápodos y 3 a 5 de arácnidos, mientras que en el bosque se registraron de 3 a 12 taxones de insectos, 1 a 5 taxones de miriápodos y 1 a 4 de arácnidos. Con respecto al tiempo de muestreo se presentaron diferencias en la riqueza de insectos y arácnidos ( $p=0,0173$, $H: 16,65 ; p=0,0020, H=17,91$ respectivamente), mientras que la riqueza de miriápodos no cambió $(p=0,3867 ; H=6,52)$. La riqueza de miriápodos y arácnidos presentaron diferencias entre los tratamientos $(p=0,0001, H=42,77 ; p=0,0001$; $\mathrm{H}=40,13$ respectivamente), mientras que la riqueza de los insectos no presentó diferencias $(p=0,5146 ; H=7,05)$. En general, la riqueza de la artropofauna edáfica no presentó diferencias entre tratamientos, pero sí presentó diferencias en el tiempo de muestreo $(p=0,0150, H=17,18)$, en julio se registró la mayor riqueza $(\bar{x}=9,67)$. En el bosque se halló mayor riqueza $(\bar{x}=12,38)$ que en el cultivo de plátano.

Se hallaron diferencias en la abundancia de insectos durante el tiempo de muestreo $(p=0,0001$; $\mathrm{H}=45,75)$, mientras que la abundancia de miriápodos y arácnidos no presentaron diferencias $(p=0,4620, H=6,25 ; p=0,4313, H=6,09$ respectivamente). Se encontró un efecto de los tratamientos sobre la abundancia de miriápodos $(p=0,0001, H=42,93)$ y arácnidos $(p=0,0001$, $\mathrm{H}=42,30$ ), mientras que la abundancia de insectos no fue afectada por los tratamientos $(p=0,5523$; $\mathrm{H}=6,85)$. Formicidae y Scarabaeidae fueron las familias más representativas en el área de estudio (Tabla 2). En general la abundancia de la artropofauna edáfica presentó diferencias en el tiempo ( $p=0,0001, H=46,40)$, siendo el mes de agosto el que presentó la abundancia más alta con 258,4 individuos en promedio.

Tan solo los insectos presentaron diferencias en la diversidad (exp Shannon) durante el tiempo de muestreo ( $p=0,0001 ; H=75,62)$ (Figura 2). Los tratamientos afectaron la diversidad de miriápodos $y$ arácnidos $(p=0,0001, H=38,65 ; p=0,0001$, $\mathrm{H}=22,07$ respectivamente), en tanto que la diversidad de insectos no presentó diferencias $(p=0,81 ; H=17,74)$. En general la diversidad de (exp Shannon) de la artropofauna edáfica presentó diferencias debidas al tiempo y al tratamiento $(p=0,0001, H=52,12 ; p=0,0026, H=23,70$ respectivamente) siendo el mes de julio el más diverso $(\bar{x}=10,07)$ y el tratamiento $N 2$ el más diverso $(\bar{x}=9,61)$; el bosque $(\bar{x}=15,64)$ es más diverso que los tratamientos en el cultivo. La dominancia de Simpson tiene el mismo comportamiento, difiere con respecto al tiempo y al tratamiento $(p=0,0001, H=55,54 ; p=0,015$, $H=18,87$ respectivamente), siendo agosto el mes con mayor dominancia $(\bar{x}=0,79)$ y el tratamiento 
A2 el más dominante $(\bar{x}=0.57)$; el bosque fue menos dominante $(\bar{x}=0,32)$.

En enero se encontró un incremento en la familia Formicidae que afectó la equitabilidad de insectos en el tiempo $(p=0,0001 ; H=78,45)$, los miriápodos $(\mathrm{p}=0,2525 ; \mathrm{H}=4,83)$ y arácnidos $(p=0,2466 ; H=2,90)$ no presentaron diferencias. A causa de los tratamientos los insectos no se ven afectados ( $p=0,6496 ; H=5,98)$, mientras que sí se presentan diferencias en la equidad de arácnidos y miriápodos $(\mathrm{p}=0,0001, \mathrm{H}=18,08$; $\mathrm{p}=0,0001, \mathrm{H}=25,58$ respectivamente) (Figura 2). En general la equidad de la artropofauna edáfica presentó diferencias debidas al tiempo y al tratamiento $(p=0,0001, H=57,56 ; p=0,0098$, $H=20,13$ respectivamente) siendo el mes de abril el más equitativo $(\bar{x}=3,42)$ y el tratamiento $N 2$ más equitativo ( $(\bar{x}=3,27)$; el bosque $(\bar{x}=4,57$ ) más equitativo que los tratamientos en el cultivo.

Por último, en el análisis de componentes principales se colapsa toda la variabilidad comunitaria en los dos primeros componentes que explican el $89 \%$. El CP1 explica el $66,2 \%$ de la variación de la comunidad, separa el bosque de los demás tratamientos en el cultivo por presentar comunidades con mayor riqueza, diversidad y equidad a las registradas en los tratamientos del cultivo. EI CP2 $(22,7 \%)$ separa las variables ambientales evaluadas. Con respecto a las variables ambientales se evidencia la correlación entre la temperatura ambiental y del suelo y entre la humedad relativa del ambiente y del suelo. Los tratamientos $\mathrm{C} 1$ y $\mathrm{C} 2$ exhiben la mayor temperatura del ambiente y suelo y el tratamiento N1 presenta la mayor humedad relativa del ambiente y suelo (Figura 3).

El análisis de componentes principales permitió visualizar el efecto de los tratamientos sobre las variables comunitarias y ambientales analizadas. Con respecto a las variables comunitarias mayor riqueza, diversidad de (exp Shannon) y equidad la presenta el tratamiento N2; la mayor abundancia y dominancia la exhiben los tratamientos $\mathrm{A} 2, \mathrm{~T} 1$, T2; y el bosque como control natural tiene la mayor riqueza, diversidad de (exp Shannon) y equidad. La mayor temperatura del ambiente y del suelo se presenta en los tratamientos $\mathrm{A} 1, \mathrm{C} 1, \mathrm{C} 2$; y la mayor humedad relativa del ambiente y el suelo la presenta el tratamiento N1.

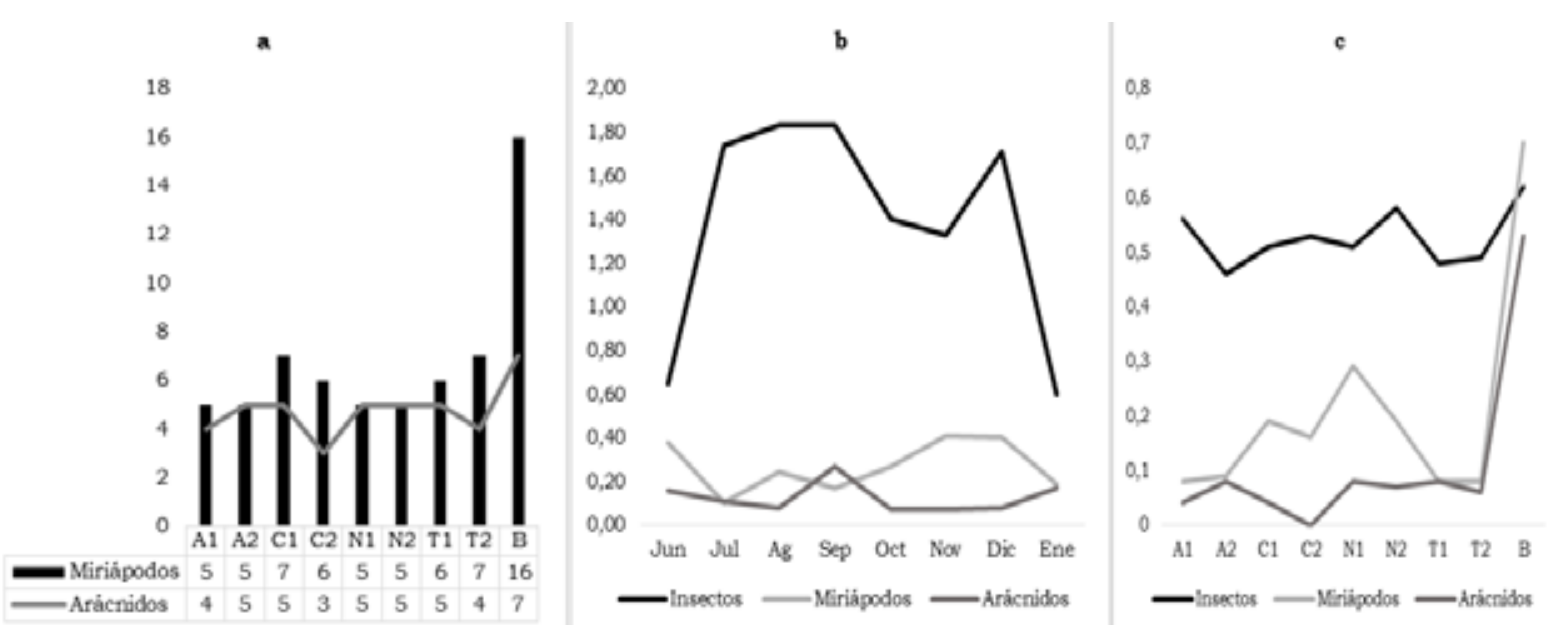

Figura 2. Modificación en la comunidad de artrópodos edáficos (por clases) a causa de los tratamientos y el tiempo de muestreo a: Variación en la riqueza especifica de miriápodos y arácnidos por los tratamientos. Tabla de datos indica riqueza específica por tratamientos. b: Variación en la diversidad de insectos, miriápodos y arácnidos durante el tiempo de muestreo (valores graficados corresponden a las medias estadísticas). c: Variación en la equidad de insectos, miriápodos y arácnidos a causa de los tratamientos (valores graficados corresponden a las medias estadísticas). A1: AGD1; A2: AGD2; C1: COD1; C2: COD2; N1: NED1; N2: NED2; T1: TD1; T2: TD2; B: bosque. jun: junio; jul: julio; ag: Agosto; sep: septiembre; oct: octubre; nov: noviembre; dic: diciembre; ene: enero. 


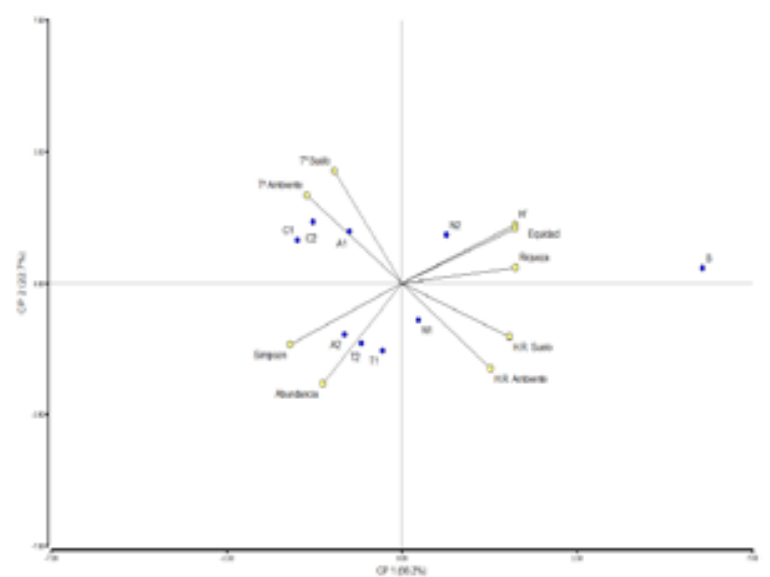

Figura 3. Variabilidad en la estructura de la comunidad de artrópodos edáficos según los tratamientos. Biplot azul (Tratamientos): A1: AGD1; A2: AGD2; C1: COD1; C2: COD2; N1: NED1; N2: NED2; T1: TD1; T2: TD2; B: JBQ. Biplot amarillo (Variables comunitarias y ambientales): Abundancia, Riqueza, $\mathrm{H}^{\prime}$ : Diversidad Shannon, Simpson: Diversidad Simpson, T Ambiente: Temperatura ambiente, To Suelo: Temperatura suelo, H.R Ambiente: Humedad relativa ambiente, H.R Suelo: Humedad relativa.

\section{Discusión}

De los organismos encontrados en este estudio, los insectos fueron los más representativos, con un $97 \%$. Las comunidades de fauna edáfica generalmente poseen riqueza baja, debido a las alteraciones causadas por la instalación y mantenimiento de los cultivos, lo cual homogeniza la estructura vegetal, como el monocultivo de plátano en la FBN. Dicho patrón se hace evidente en el presente estudio, ya que de manera general la riqueza de la comunidad de artrópodos edáficos en los tratamientos del cultivo en la FBN no difiere entre sí, mientras esta riqueza si difiere en el bosque secundario.

La abundancia alta de hormigas y escarabajos coincide con lo reportado para en sistemas productivos en otras investigaciones, donde estos individuos representan el mayor número respecto a los demás taxones hallados, así, COTES et al. (2010), JEREZ-VALLE et al. (2014). TURNER y FOSTER (2009) encontraron que las poblaciones de hormigas y escarabajos permanecen en los sistemas productivos y CARRIERE et al. (2009), han demostrado que las poblaciones de hormigas y escarabajos en sistemas semi-naturales se asocian a sistemas productivos con prácticas inadecuadas.

El predominio de Formicidae y Scarabaeidae está relacionado con la oferta de recursos orgánicos, lo que probablemente favoreció la abundancia de estos insectos. Según NEHER (1999), las plantas y los desechos orgánicos proveen el hábitat y recurso alimenticio a los organismos del suelo. Asimismo, CABRERA et al. (2011) resaltan que Formicidae y Coleóptera son, generalmente, los grupos más abundantes y frecuentes en cultivos de importancia agrícola, debido a las adaptaciones $y$ amplios rangos de movimiento que tienen estos organismos. Además, MUÑOZ y VILLOTA (2014) señalan que las hormigas por sus hábitos generalistas, pueden disponer de un amplio rango de recursos alimenticios.

La artropofauna hallada en la hojarasca tan solo aporta el $2,1 \%$ de los individuos colectados, haciendo evidente un proceso de perturbación sobre el sistema edáfico debido a las prácticas de manejo del cultivo en la FBN, mientras que la condición del bosque secundario del JBQ favorece la presencia de consumidores primarios, como arácnidos y miriápodos, en este sustrato. Se conoce la susceptibilidad alta de los arácnidos e insectos edáficos de hábitos saprófagos y zoófagos (ejemplo: Scarabaeidae, Elateridae, Staphylinidae, Cydnidae y Pyralidae) a la perturbación (GÓMEZ et al., 2016), como lo demuestran las diferencias encontradas en las dos áreas de trabajo de nuestro estudio. Con relación a lo anterior, LANG-OVALLE et al., (2011) y DE LA ROSA y NEGRETE-YANKELEVICH (2012) atribuyen dicho comportamiento al aporte arbóreo heterogéneo de bosques secundarios como el del JBQ, debido a que la macrofauna edáfica en bosques conservados se encuentra preferentemente en la hojarasca.

Con relación a la homogeneidad entre la comunidad de insectos edáficos presentes en los diferentes tratamientos, LANG-OVALLE et al., (2011) y TESSARO et al. (2013) observan que la riqueza y la diversidad de la macrofauna edáfica no presenta diferencias en los distintos niveles de fertilización a la cual se someten los sistemas agroproductivos, debido a la capacidad adaptativa, ciclos de vida 
cortos, tasas altas de reproducción y la presencia de macroagregados que son consumidos por comunidades edáficas. La homogeneidad de los sistemas de monocultivo es un factor determinante para la artropofauna, debido a que la riqueza vegetal influye directamente sobre la riqueza y abundancia de comunidad edáfica (TURNER y FOSTER, 2009; GHALAZI et al., 2016).

La baja diversidad encontrada en los tratamientos en el cultivo con respecto al bosque, se puede explicar por las prácticas agrícolas, tales como, la labranza del suelo, el plateo, la aplicación de plaguicidas, las cuales tiene un efecto directo sobre la diversidad de la artropofauna asociada, pues estos procesos, pueden alterar la textura del suelo, principal recurso para la reproducción de muchas especies (GHAZALI et al., 2016; LAGERÖF et al., 2017).

Algunos autores (BARNES et al., 2014; GKISAKIS et al., 2015; LAGERLÖF et al., 2017) han encontrado que existe disminución de la diversidad de la artropofauna en un valor cercano al $45 \%$ en una transición bosque - sistema productivo, puesto que la variación en la temperatura ambiente, del suelo y la humedad relativa en la transición bosque sistema productivo afecta la diversidad de artropofauna, con tendencia a la homogeneización debido a la ausencia de depredadores naturales, quienes necesitan de condiciones ambientales específicas.

La modificación de la comunidad de arácnidos en el cultivo de plátano de la FBN se debe posiblemente a las prácticas de manejo utilizadas en este cultivo durante períodos de producción de plátano, superiores a 10 años, que homogenizaron la estructura vegetal, quedando expuesto el sistema edáfico a las altas temperaturas, reduciendo así la disponibilidad de nicho y refugio para comunidades epigeas. TESSARO et al. (2013) resaltan que en sistemas agro-productivos, la baja diversidad de arácnidos es consecuencia de la utilización de prácticas agrícolas y de gestión del suelo inadecuadas, como la aplicación de plaguicidas. Además, LO-MAN-HUNG et al. (2011) evidenciaron que el manejo agrícola de uso del suelo impacta la comunidad de arácnidos, disminuyendo su riqueza y diversidad, especialmente de familias que buscan activamente sus presas como Gnaphosidae y Lycosidae, que para el presente trabajo no representaron más del $20 \%$ de individuos colectados en los tratamientos del cultivo en la FBN.

La variación en la abundancia de Formicidae posiblemente se relaciona con el impacto de la instalación del cultivo de plátano que causa la perdida de cobertura vegetal, lo cual expone al suelo a condiciones climáticas extremas, como la temperatura ambiente alta $\left(>30^{\circ} \mathrm{C}\right)$ y la perdida de humedad relativa en el suelo $(<20 \%)$. Esto favorece el incremento de la mimercofauna, que son indicadores de las áreas que han sufrido modificaciones sustanciales por la acción antrópica, la gestión del suelo y la contaminación industrial, donde la afectación y la distribución de la comunidad en los suelos tropicales depende de la duración de la época seca y la capacidad del suelo para retener agua (LAVELLE et al., 2012; ANDERSEN et al., 2002 y LANG-OVALLE et al., 2011).

La abundancia baja de miriápodos y su estabilidad en el tiempo de muestreo, a pesar de las variaciones microclimáticas, se atribuye, según RIUTTA et al., (2012), a los bajos rangos de dispersión, adaptaciones fisiológicas y de comportamiento de estos organismos, que construyen refugios subterráneos para soportar periodos de sequía, y posteriormente, cuando las condiciones de humedad se restablecen por los periodos de lluvias, los organismos se activan y se mantienen en el tiempo. Además, GAMBOA-TABARES et al. (2010) atribuyen la baja abundancia de miriápodos en los cultivos a la poca cantidad de biomasa (hojarasca y mantillo) lugares que habitan.

\section{Conclusiones}

El presente estudio muestra que la riqueza de la comunidad de artrópodos edáficos no se ve afectada por los tratamientos utilizados en el cultivo de plátano de la FBN.

La cobertura vegetal mixta que presenta el bosque secundario JBQ favorece la diversidad alta de la comunidad de artrópodos edáficos que difiere de la comunidad homogénea presente en el cultivo que se ven afectada por el aumento desproporcionado de la familia Formicidae. 
El registro exclusivo de familias de miriápodos (Ballophilidae, Oryidae, Mecistocephalidae, Pseudonannolenidae, Aphelidesmidae y Stemmiulidae) y arácnidos (Pholcidae y Hubbardiidae) en el bosque secundario JBQ se ve favorecido por el aporte de biomasa vegetal y la presencia de artrópodos asociados que favorecen el desarrollo de comunidades de hábitos fitófagos y zoófagos.
Agradecimientos. Este trabajo fue financiado por el Departamento de Ciencia y Tecnología de Colombia (COLCIENCIAS) y el Banco Interamericano de Desarrollo (BID), Grant \# 1113545-31135, RC 551 of 2012; La Gobernación del Quindío y la Universidad del Quindío (proyecto interno \# 582). A la Asociación de productores de plátano del Quindío MUSACEAS y al Jardín Botánico del Quindío.

\section{Referencias}

ANDERSON, J.M.; INGRAM, J.S.I. 1993. Tropical soil biology and fertility: a handbook of methods. 2 edition, C.A.B. Oxford University Press. Oxford.

BORROR, D.J.; WHITE, R. 1970. A field guide to insetcs America north of Mexico. Houghton Mifflin Company. Boston.

BARNES, A.D.; JOCHUM, M.; MUMME, S.; HANEDA, N.F.; FARAJALLAH, A.; WIDARTO, T.H.; BROSE, U. 2014. Consequences of tropical land use for multitrophic biodiversity and ecosystem functioning, Nature Communications: 1-7. DOI: 10.1038/ncomms6351.

CABRERA, G.; ROBAINA, N.; LEON, P. 2011. Riqueza y abundancia de la macrofauna edafica en cuatro usos de la tierra en las provincias de Artemisa y Mayabeque, Cuba. Pastos y Forrajes 34 (3):313-330.

CARRIERE, Y.; ELLERS-KIRK, C.; CATTANEO, M.G.; YAFUSO, C.M.; ANTILLA, L.; HUANG, C.Y.; RAHMAN, M.; ORR, B.J.; MARSH, S.E. 2009. Landscape effects of transgenic cotton on non-target ants and beetles. Basic Applied Ecology 10:597-606.

CHACÓN DE ULLOA, P.; OSORIO-GARCÍA, A.; ACHURY, R.; BERMÚDEZ- RIVAS, C. 2012. Hormigas (Hymenoptera: Formicidae) del bosque seco tropical (Bs-T) de la cuenca alta del río Cauca, Colombia. Biota Colombiana 13(2):165-181.

CHOATE, P.M. 2010. Introduction to identification of adult insects and related Arthropods. Disponibñe en: http://entnemdept.ifas.ufl.edu/choate/arthropod id.pdf. Consultado Abril 15, 2013.

COTES, B.; CAMPOS, M.; PASCUAL, F.; GARCÍA, P.A.; RUANO, F. 2010. Comparing taxonomic levels of epigeal insects under different farming systems in andalusian olive agroecosystems. Applied Soil Ecology 44:228-236.

COLEMAN, D.; CROSSLEY, D.; HENDRIX, P. 2004. Fundamentals of soil ecology. Elsevier Academic Press. Burlington, MA.

CORPORACIÓN AUTÓNOMA REGIONAL DEL QUINDÍO (CRQ). 2014. Sistema de información geográfico. Disponiblñe en: http://200.21.93.53/sigquindioii/VisorGeneral.aspx. Consultado Abril 1, 2014.

COLWELL, R.K. 1997. Estimates: Statistical Estimation of Species Richness and Shared Species from Samples (Software and User's Guide), Versión 5.0.1. Disponible en: http://viceroy.eeb.uconn.edu/ estimates/. Consultado Abril 1, 2014. 
DECAENS, T.; ASAKAWA, N.; GALVIS, J.H.; THOMAS, R.J.; AMEZQUITA, E. 2003. Surface activity of soil ecosystem engineers and soil structure in contrasted land use systems of Colombia. European Journal of Soil Biology 38:267-271.

DE LA ROSA, I.N.; NEGRETE-YANKELEVICH, S. 2012. Distribución espacial de la macrofauna edafica en bosque mesofilo, bosque secundario y pastizal en la reserva La Cortadura, Coatepec, Veracruz, México. Revista Mexicana de Biodiversidad 83:201-215.

DELVARE, G.; ABERLENC, H.P.; MICHEL, B.; FIGUEROA, A. 2002. Los insectos de África y América Tropical, clave para la identificación de las principales familias. CIRAD.

DI RIENZO, J.A.; CASANOVES, F.; BALZARINI, M.G.; GONZÁLEZ. L.; TABLADA M.; ROBLEDO, C.W. InfoStat versión 2016. Grupo InfoStat, FCA, Universidad Nacional de Córdoba, Argentina. URL http:// www.infostat.com.ar. Consultado Abril 1, 2014.

FEIJOO, A.; ZÙÑIGA M.C.; QUINTERO, H.; LAVELLE, P. 2007. Relaciones entre el uso de la tierra y las comunidades de lombrices en la cuenca del rio La Vieja, Colombia. Pastos y Forrajes 30(2):235-249.

FEIJOO, A.; ZÙÑIGA, M.C.; QUINTERO, H.; CARVAJAL-VANEGAS, A.F.; ORTIZ, D.P. 2010. Patrones de asociación entre variables del suelo y usos del terreno en la cuenca del rio la vieja, Colombia. Acta Zoologica Mexicana 2:151-164.

GAMBOA-TABARES, J.A.; ORJUELA, J.A.; MARTİNEZ, L.L.; MUÑOZ, F.A. 2010. Macroinvertebrados edáficos asociados a tres tipos de cobertura en paisaje de Lomerío (Caquetá, Colombia). Ingeniería y Amazonía 3(1):5-15.

GHAZALI, A.; ASMAH, S.; SYAFIQ, M.; YAHYA, M.S.; AZIZ, N.; PENG, T.L.; NORHISHAM, H.R.; PUAN, C.L.; TURNER, E.C.; AZHAR, B. 2016. Effects of monoculture and polyculture farming in oil palm smallholdings on terrestrial arthropod diversity. Journal of Asia-Pacific Entomology 19:415- 421.

GIBB, T.J.; OSETO C.Y. 2006. Arthropod collection and identification. Aca. Pre. Serv. USA.

GKISAKIS, V.; VOLAKAKIS, N.; KOLLAROS, D.; BÀRBERI, P.; KABOURAKIS, E.M. 2016. Soil Arthropod community in the olive agroecosystem: Determined by enviroment and farming practices in different management system and agroecological zones. Agriculture, Ecosystems and Enviroment. 218:178 189.

GÓMEZ, D.F.; GODOY, M.C.; CORONEL J.M. 2016. Macrofauna edáfica en ecosistemas naturales y agroecosistemas de la ecoregión esteros del Iberá (corrientes, argentina). Cienc Suelo (Argentina) 34(1):43-56.

HOLDRIDGE, L. 1987. Ecología basada en zonas de vida. IICA. San José, Costa Rica.

JEREZ-VALLE, C.; GARCÍA, P.A.; CAMPOS, M.; PASCUAL, F. 2014. A simple bioindication method to discriminare olive orchad management types using the soil arthropod fauna. Applied Soil Ecology 76:42 -51.

LAGERLÖF, J.; MARIBIE, C.; JOHN, J.M. 2017. Trophic interactions among soil arthropods in contrasting land-use systems in Kenya, studied with stable isotopes. European Journal of Soil Biology 79:31-39.

LANG-OVALLE, F.P.; PEREZ-VÁZQUEZ, A.; MARTÍNEZ-DÁVILA, J.P.; PLATAS-ROSADO, D.E.; OJEDAENCISO, L.A.; GONZÁLEZ-ACUÑA, I.J. 2011. Macrofauna edáfica asociada a plantaciones de mango y caña de azúcar. Terra Latinoamericana 29(2):169-177. 
LAVELLE, P. 2012. Soil as a habitat. Págs. 7-27. En: Wall, D.H. (ed). Soil ecology and ecosystem service. Oxford. Oxford University Press.

LOU, J.; GONZÁLEZ-OREJA, J. 2012. Midiendo la diversidad biológica: más allá del índice de Shannon. Acta zoológica Lilloana 56(1-2):3-14.

LO-MAN-HUNG, N.F.; MARICHAL, R.; CANDIANI, D.F.; CARVALHO, L.S.; INDICATTI, R.P.; BONALDO, A.B.; COBO, D.H.; FEIJOO, A.; TSELOUIKO, S.; PRAXEDES, C.; BROWN, G.; VELAZQUES, E.; DECAENS, T.; OSZWALD, J.; MARTINS, M.; LAVELLE, P. 2011. Impact of different land management on soil spiders (Arachnida: Araneae) in two Amazonian áreas of Brasil and Colombia. Journal of Arachnology 39:296-302.

MUÑOZ, S.; VILLOTA, T. 2014. Evaluación de la macrofauna y composición florística en sistemas productivos de café (Coffea arabica L.), municipio La Unión Nariño. (Disertación de pregrado, Facultad de Ciencias Agropecuarias Universidad de Nariño. Pasto.

NEHER, D.A. 1999. Soil community and ecosystem proceses; comparing agricultural ecosystems with natural ecosystems. Agrofor Systems 45:159-185.

RITZ, K.; VAN DER PUTTEN, W. 2012.The living soil and ecosystem services. P'gas. 5-6. En: Wall, D.H. (ed.). Soil ecology and ecosystem service. Oxford. Oxford University Press.

RIUTTA, T.; SLADE, E.; BEBBER, D.P.; TAYLOR, M.E.; MALHI, Y.; RIORDAN, P.; MACDONALD, D.W.; MORECROFT, M.D. 2012. Experimental evidence for the interacting effect of forest edge, moisture and soil macrofauna on leaf litter decomposition. Soil biology and Biochemistry 49:124-131.

SWIFT, M.J.; HEAL, W.; ANDERSON, M. 1979. Decomposition in terrestrial ecosystems. Blackwell Scienctific Publication. London, UK

TESSARO, D.; SAMPAIO, S.C.; ALVES, L.F.; DIETER, J.; CORDOVIL, C.M.; VARENNES, A.; PANSERA, A. 2013. Macrofauna of soil treated with swine wastewater combined with chemical fertilization. African Journal of Agricultural Research 8(1):86- 92.

TURNER, E.C.; FOSTER, W.A. 2009. The impact of forest conversion to oil palm on arthropods abundance and biomass in Sabah, Malaysia. Journal Of Tropical Ecology. 25:23-30.

WOLFF, E.M. 2006. Insectos de Colombia, Guía básica de familias. GIEM. Univ. Ant. Medellín, Colombia.

ZUÑIGA, C.; FEIJOO, A.; QUINTERO, H.; ALDANA, N.; CARVAJAL A. 2013 Farmers' perceptions of earthworms and their role in soil. Applied Soil Ecology 69:61-68. 